[9] A. S. Matveev and A. V. Savkin, "Stabilization of multisensor networked control systems with communication constraints," in Proc. Asian Contr. Conf., Melbourne, Australia, July 2004.

[10] S. Tatikonda, "Some scaling properties of large distributed control systems," in Proc. 42nd IEEE Conf. Dec. Contr., Maui, Hawaii, Dec. 2003, pp. 3142-3147.

[11] G. N. Nair, R. J. Evans, and P. E. Caines, "Stabilising decentralised linear systems under data rate constraints," in Proc. 43rd IEEE Conf. Dec. Contr., Bahamas, Dec. 2004, pp. 3992-3997.

[12] S. Yüksel, "State Estimation and Control for LTI Systems over Communication Channels,", University of Illinois at Urbana-Champaign, Urbana-Champaign, IL, Oct. 2003, M.S. thesis.

[13] S. Yüksel and T. Başar, "On the absence of rate loss in decentralized sensor and controller structure for asymptotic stability," in Proc. IEEE Amer. Contr. Conf., Minneapolis, MN, Jun. 2007.

[14] S. Yüksel and T. Başar, "Decentralized multi-controller stabilizability over communication channels," in Proc. European Contr.Conf., Kos, Greece, Jun. 2007.

[15] J. D. Slepian and J. K. Wolf, "Noiseless coding of correlated information sources," IEEE Trans. Inf. Theory, vol. 19, pp. 471-480, Jul. 1973.

[16] S. S. Pradhan and K. Ramchandran, "Distributed source coding using syndromes (DISCUS): Design and construction," IEEE Trans. Inf. Theory, vol. 49, pp. 626-643, Mar. 2003.

[17] J. Kusuma, L. Doherty, and K. Ramchandran, "Distributed compression for sensor networks," in Proc. IEEE Conf. Image Processing, Thessaloniki, Greece, Oct. 2001.

[18] A. D. Wyner and J. Ziv, "The rate-distortion function for source coding with side information at the decoder," IEEE Trans. Inf. Theory, vol. 22, no. 1, pp. 1-11, Jan. 1976.

[19] S. Tatikonda, A. Sahai, and S. Mitter, "Stochastic linear control over a communication channel," IEEE Trans. Autom. Control, vol. 49, no. 9, pp. 1549-1561, Sep. 2004.

\section{On the Gap Between Positive Polynomials and SOS of Polynomials}

Graziano Chesi, Senior Member, IEEE

\begin{abstract}
This note investigates the gap existing between positive polynomials and sum of squares (SOS) of polynomials, which affects several analysis and synthesis tools in control systems based on polynomial SOS relaxations, and about which almost nothing is known. In particular, a matrix characterization of the PNS, that is the positive homogeneous forms that are not SOS, is proposed, which allows to show that any PNS is the vertex of an unbounded cone of PNS. Moreover, a complete parametrization of the set of PNS is introduced.
\end{abstract}

Index Terms-Hilbert's 17th problem, linear matrix inequality (LMI), optimization, positive polynomial, sum of squares (SOS).

\section{INTRODUCTION}

Positive polynomials play a key role in the analysis and synthesis of control systems. This is due to the fact that conditions for establishing stability of equilibrium points or computing performance indexes of the system such as the $\mathcal{H}_{\infty}$ norm, can be reformulated in terms of positivity of a Lyapunov function and negativity of its time derivative. These functions are usually polynomials as natural extension of the classic quadratic Lyapunov functions in the attempt of achieving less conservative results. Unfortunately, establishing whether a polynomial is positive or not, is still a difficult problem that cannot be solved systematically because it amounts to solving a nonconvex optimization. In order to deal with this problem, gridding methods have been proposed, for example based on the use of Chebychev points, but their conservativeness and computational burden are generally unacceptable, reason that has motivated the search for alternative approaches.

This search has recently provided the sum of squares (SOS) relaxation (among the first contributions on SOS relaxation, see for example [1]). In this approach, the positivity of a homogeneous form (equivalently of a polynomial) is established by checking if it is a SOS of homogeneous forms, operation which amounts to solving a linear matrix inequality (LMI) feasibility problem, i.e., a convex optimization. Due to the existence of powerful tools for solving LMIs [2], SOS relaxations have quickly become an essential tool in control. In robust control, SOS relaxations have been employed to obtain less conservative conditions than those provided by quadratic Lyapunov functions to assess robust stability of linear systems affected by parametric uncertainty, in both cases of time-varying uncertainty [3]-[6] and time-invariant uncertainty [7]-[9]. An analogous use of SOS has been made in the computation of robust performance indexes [10], [11]. SOS have been exploited also in the field of nonlinear systems [12]-[15], hybrid systems [16], [17] and time-delay systems [18]. See also [19]-[22] for further applications of SOS.

"Can any positive homogeneous form be written as a SOS?" This question was made by Hilbert in his 17 th problem and has a negative answer as it is known. It is hence known that, in spite of their popularity, SOS relaxations can be conservative. However, almost nothing

Manuscript received February 9, 2005; revised December 1, 2006. Recommended by Associate Editor M. Fujita.

The author is with the Department of Electrical and Electronic Engineering, University of Hong Kong, Pokfulam Road, Hong Kong (e-mail chesi@eee.hku. hk).

Digital Object Identifier 10.1109/TAC.2007.899083 
TABLE I

NOTATION FOR HOMOGENEOUS FORMS

\begin{tabular}{|l|l|}
\hline$\Xi_{n, m}:$ set of homogeneous forms $f(x)$ of degree $m$ in $x \in \mathbb{R}^{n}$ & $x^{[m]} \in \mathbb{R}^{\sigma(n, m)}$ : base for $\Xi_{n, m}$ satisfying (6) \\
\hline$\sigma(n, m)=((n-1) ! m !)^{-1}(n+m-1) !$ & $\tau(n, 2 m)=\frac{1}{2} \sigma(n, m)[\sigma(n, m)+1]-\sigma(n, 2 m)$ \\
\hline$\mu(g)=\min _{x} g(x)$ s.t. $\|x\|=1 \quad$ (positivity index) & $\lambda(g)=\max _{\alpha} \lambda_{\min }(G+L(\alpha)) \quad$ (SOS index) \\
\hline$\Phi_{n, 2 m}:$ set of positive forms in $\Xi_{n, 2 m}$ & $g(x) \in \Phi_{n, 2 m} \Longleftrightarrow \mu(g) \geq 0$ \\
\hline$\Sigma_{n, 2 m}:$ set of SOS in $\Xi_{n, 2 m}$ & $g(x) \in \Sigma_{n, 2 m} \Longleftrightarrow \lambda(g) \geq 0$ \\
\hline$\Delta_{n, 2 m}:$ set of PNS in $\Xi_{n, 2 m}$ & $g(x) \in \Delta_{n, 2 m} \Longleftrightarrow \mu(g) \geq 0$ and $\lambda(g)<0$ \\
\hline
\end{tabular}

is presently known about the set of homogeneous forms that are positive but not SOS (we will refer to such homogeneous forms as PNS). See [23] for a survey on this problem.

The aim of this note is to characterize PNS since actually they represent the gap between several fundamental problems in control systems and the corresponding solution tools. First, some remarks about the distance between PNS and SOS are introduced, in particular showing that the set of PNS, when not empty, has a non empty interior. Then, a matrix characterization of PNS is proposed based on eigenvectors and eigenvalues decomposition. This characterization is based on the concept introduced in this paper of maximal matrix for the representation of homogeneous forms. It is shown that any PNS is the vertex of an unbounded cone of PNS whose directions correspond to strictly positive SOS. This cone can be linearly parameterized in a convex set. Moreover, a complete parametrization of the set of PNS is proposed, providing hence a technique to construct PNS.

This note is organized as follows. Section II introduces some preliminaries about the representation and classification of homogeneous forms. Section III presents the main results of the paper about the representation of homogeneous forms and characterization of PNS. Last, Sections IV and V conclude with some illustrative examples and remarks.

\section{PRELIMINARIES}

\section{A. Homogeneous Forms Representation}

Let us define the following notation: $\mathbb{N}, \mathbb{R}$ : natural number set (including 0 ) and real number set; $\mathcal{S}_{n}$ : set of symmetric matrices $n \times n ; I_{n}$ : identity matrix $n \times n ; A^{\prime}$ : transpose of matrix $A ; A \succ 0(A \succeq 0)$ : symmetric positive definite (semidefinite) matrix $A ; \lambda_{\min }(A):$ minimum real eigenvalue of $A ; \operatorname{ker}(A)$ : null space of matrix $A ; \operatorname{img}(A)$ : image of matrix $A$; $\operatorname{diag}(x)$ : diagonal matrix $n \times n$ whose diagonal components are the components of $x \in \mathbb{R}^{n} ; x_{i}$ or $(x)_{i}: i$-th component of vector $x ; x \geq 0(x>0)$ : vector with positive (strictly positive) components; $x^{q}: x_{1}^{q_{1}} x_{2}^{q_{2}} \cdots x_{n}^{q_{n}}$ with $x \in \mathbb{R}^{n}, q \in \mathbb{N}^{n}$; s.t.: subject to.

We say that $f(x)$ is a homogeneous form of degree $m$ in $x \in \mathbb{R}^{n}$ if

$$
f(x)=\sum_{q \in \mathcal{Q}_{n, m}} c_{q} x^{q}
$$

where $c_{q} \in \mathbb{R}$ are the coefficients of $f(x)$ and $\mathcal{Q}_{n, m}$ is the index set

$$
\mathcal{Q}_{n, m}=\left\{q \in \mathbb{N}^{n}: \sum_{i=1}^{n} q_{i}=m\right\}
$$

with cardinality equal to $\sigma(n, m)$ defined in Table I. The set of homogeneous forms of degree $m$ in $x \in \mathbb{R}^{n}$ is denoted by $\Xi_{n, m}$. Let $x^{[m]} \in \mathbb{R}^{\sigma(n, m)}$ be a vector whose components constitute a base for the homogeneous forms in $\Xi_{n, m}$. For any $f(x) \in \Xi_{n, m}$ we define the norm

$$
\|f(x)\|_{c}=\|\vec{f}\| \text { where } \vec{f}^{\prime} \text { is such that } f(x)=\vec{f}^{\prime} x^{[m]} \text {. }
$$

Any $g(x) \in \Xi_{n, 2 m}$ can be written as

$$
g(x)=x^{[m]}(G+L(\alpha)) x^{[m]}
$$

where $G \in \mathcal{S}_{\sigma(n, m)}$ is any matrix satisfying $g(x)=x^{[m]} \mathrm{Gx}^{[m]}$ and $L(\alpha)$ is any linear parametrization of the set

$$
L_{n, 2 m}=\left\{L \in \mathcal{S}_{\sigma(n, m)}: x^{[m]} L x^{[m]}=0\right\}
$$

with dimension equal to $\tau(n, 2 m)$ defined in Table I. The representation (4) is known as complete square matricial representation (CSMR) (see [1], [20]) and Gram matrix method [23]. In the sequel we will say that the matrix $G$ (resp., $G+L(\alpha)$ ) in (4) is a SMR (resp., CSMR) matrix of $g(x)$. In the sequel we suppose that $x^{[m]}$ satisfies

$$
x^{[m]} x^{[m]}=\left(\sum_{i=1}^{n} x_{i}^{2}\right)^{m} .
$$

A possible choice guaranteeing this property is obtained by defining the $i$-th component of $x^{[m]} \in \mathbb{R}^{\sigma(n, m)}$ as

$$
\left(x^{[m]}\right)_{i}=\sqrt{\frac{m !}{(\varphi(i))_{1} !(\varphi(i))_{2} ! \cdots(\varphi(i))_{n} !}} x^{\varphi(i)} .
$$

where $\varphi:\{i \in \mathbb{N}: 1 \leq i \leq \sigma(n, m)\} \rightarrow \mathcal{Q}_{n, m}$ is any bijective function.

\section{B. Positive Forms, SOS and PNS}

We say that $g(x) \in \Xi_{n, 2 m}$ is positive if $g(x) \geq 0$ for all $x$ or, equivalently, if $\mu(g) \geq 0$ where $\mu(g)$ is the positivity index of $g(x)$ in Table I (observe in fact that the positivity of $g(x)$ does not depend on the norm of $x$ ).

The form $g(x) \in \Xi_{n, 2 m}$ is a SOS if and only if there exist $f_{1}(x), \cdots, f_{k}(x) \in \Xi_{n, m}$ such that

$$
g(x)=\sum_{i=1}^{k} f_{i}(x)^{2}
$$

It is straightforward to verify that $g(x)$ is a SOS if and only if there exists $\alpha$ such that $G+L(\alpha) \succeq 0$ or, equivalently, if and only if $\lambda(g) \geq$ 
0 where $\lambda(g)$ is the SOS index of $g(x)$ in Table I. This index can be computed by solving the eigenvalue problem (EVP)

$$
\lambda(g)=\max _{t, \alpha} t \text { s.t. } G+L(\alpha)-t I_{\sigma(n, m)} \succeq 0
$$

that is a convex optimization constrained by LMIs.

The form $g(x) \in \Xi_{n, 2 m}$ is a PNS if and only if $g(x)$ is positive but it is not a SOS or, equivalently, if and only if $\mu(g) \geq 0$ and $\lambda(g)<0$.

We denote the sets of positive forms, SOS, and PNS in $\Xi_{n, 2 m}$ as $\Phi_{n, 2 m}, \Sigma_{n, 2 m}$ and $\Delta_{n, 2 m}$, respectively. These sets clearly satisfy

$$
\begin{aligned}
& \Xi_{n, 2 m} \supset \Phi_{n, 2 m}, \Phi_{n, 2 m}=\Sigma_{n, 2 m} \cup \Delta_{n, 2 m}, \\
& \Sigma_{n, 2 m} \cap \Delta_{n, 2 m}=\emptyset .
\end{aligned}
$$

It has been shown that $\Delta_{n, 2 m}$ is empty in the following cases [23], [24]:

- $m=1$ for all $n$;

- $n \leq 2$ for all $m$;

- $n=3$ and $m \leq 2$.

\section{MAIN RESULTS}

\section{A. The Maximal SMR Matrix}

Let us introduce the following concept, which is the base for the characterization of PNS proposed in this paper. Given $g(x) \in \Xi_{n, 2 m}$, a SMR matrix $G$ of $g(x)$ is said maximal if

$$
\lambda_{\min }(G)=\lambda(g)
$$

The maximal SMR matrices of $g(x)$ are hence given by

$$
G+L\left(\alpha^{*}\right)
$$

where $\alpha^{*}$ is any optimal value of $\alpha$ in (9) ( $\alpha^{*}$ exists because $\lambda(g)$ is bounded whenever $\|g(x)\|_{c}$ is bounded).

In order to characterize the maximal SMR matrices, let us introduce the following definition. The quadruplet $\left\langle\lambda_{\min }(G), \beta, V_{0}, V_{p}\right\rangle$ is said a decomposition of matrix $G \in \mathcal{S}_{\sigma(n, m)}$ if

$$
G=V D V^{\prime}
$$

where $D \in \mathcal{S}_{\sigma(n, m)}$ is the diagonal matrix containing the eigenvalues of $G$ defined by the minimum eigenvalue $\lambda_{\text {min }}(G)$ of multiplicity $\sigma(n, m)-r$ and the vector $\beta \in \mathbb{R}^{r}, \beta>0$, as

$$
D=\lambda_{\min }(G) I_{\sigma(n, m)}+\left[\begin{array}{ll}
0 & \\
& \operatorname{diag}(\beta)
\end{array}\right]
$$

and $V \in \mathbb{R}^{\sigma(n, m) \times \sigma(n, m)}$ is an orthonormal eigenvector matrix defined as

$$
V=\left[V_{0} V_{p}\right]
$$

where the columns of $V_{0} \in \mathbb{R}^{\sigma(n, m) \times \sigma(n, m)-r}$ are a base of the eigenspace of the minimum eigenvalue, and the columns of $V_{p} \in \mathbb{R}^{\sigma(n, m) \times r}$ are bases for the eigenspaces of the other eigenvalues.

Let us observe that the introduced decomposition is not unique. In fact, there are multiple choices for $\beta, V_{0}, V_{p}$ that satisfy the required conditions. In particular, if $\left\langle\lambda_{\min }(G), \beta, V_{0}, V_{p}\right\rangle$ is a decomposition of
$G$, it follows that also $\left\langle\lambda_{\min }(G), B \beta, V_{0} A, V_{p} B\right\rangle$ is for all orthonormal matrices $A \in \mathbb{R}^{\sigma(n, m)-r \times \sigma(n, m)-r}$ and for all permutation matrices $B \in \mathbb{R}^{r \times r}$.

The following result holds.

Theorem 1: Let $\left\langle\lambda_{\min }(G), \beta, V_{0}, V_{p}\right\rangle$ be any decomposition of the matrix $G \in \mathcal{S}_{\sigma(n, m)}$ and define

$$
\zeta\left(V_{0}\right)=\max _{\|\alpha\|=1} \lambda_{\min }\left(V_{0}^{\prime} L(\alpha) V_{0}\right) .
$$

Then, $G$ is a maximal SMR matrix if and only if $\zeta\left(V_{0}\right) \leq 0$. Moreover, $\zeta\left(V_{0}\right)$ does not depend on the chosen decomposition.

Proof: From (11) it follows that $G$ is maximal if and only if

$$
\lambda_{\text {min }}(G+L(\alpha)) \leq \lambda_{\min }(G) \forall \alpha \neq 0
$$

and, hence, if and only if for all $\alpha \neq 0$ there exists $y \in \mathbb{R}^{\sigma(n, m)},\|y\|=$ 1 , such that

$$
y^{\prime}(G+L(\alpha)) y \leq \lambda_{\min }(G) .
$$

Let $\left\langle\lambda_{\min }(G), \beta, V_{0}, V_{p}\right\rangle$ be a decomposition of $G$. Then, (17) can be rewritten as

$$
y^{\prime} V_{p} \operatorname{diag}(\beta) V_{p}^{\prime} y \leq-y^{\prime} L(\alpha) y .
$$

Observe that $L(\alpha)$ depends linearly on $\alpha$. This means that $V_{p}^{\prime} y$ must tend to zero as $\alpha$ tends to zero $\operatorname{since} \operatorname{diag}(\beta) \succ 0$. Moreover, if (18) holds with the pair $\langle y, \alpha\rangle$, it also holds with the pair $\langle y, c \alpha\rangle$ for all $c \geq 1$. Therefore, it turns out that $G$ is maximal if and only if

$$
\forall \alpha \neq 0 \forall \varepsilon>0 \exists y,\|y\|=1:\left\|V_{p}^{\prime} y\right\|<\varepsilon \quad \text { and (18) holds }
$$

or, equivalently, if and only if

$$
\forall \alpha \neq 0 \exists y,\|y\|=1: V_{p}^{\prime} y=0 \text { and (18) holds. }
$$

Since $\operatorname{ker}\left(V_{p}^{\prime}\right)=\operatorname{img}\left(V_{0}\right)$, it follows that $V_{p}^{\prime} y=0$ if and only if $y \in \operatorname{img}\left(V_{0}\right)$. Hence, (19) can be rewritten as

$$
\forall \alpha \neq 0 \exists y \in \operatorname{img}\left(V_{0}\right),\|y\|=1: y^{\prime} L(\alpha) y \leq 0 .
$$

Write $y \in \operatorname{img}\left(V_{0}\right)$ as $y=V_{0} p$ with $p \in \mathbb{R}^{\sigma(n, m)-r}$. Since $y^{\prime} L(\alpha) y$ depends linearly on $\alpha$, condition (20) can be rewritten as

$$
\forall \alpha,\|\alpha\|=1, \exists p,\|p\|=1: p^{\prime} V_{0}^{\prime} L(\alpha) V_{0} p \leq 0
$$

and, hence, as $\zeta\left(V_{0}\right) \leq 0$.

Last, observe that the choice of $V_{0}$ in the decomposition of $G$ does not affect $\zeta\left(V_{0}\right)$. In fact, all the matrices whose columns are an orthonormal base of the eigenspace of the minimum eigenvalue of $G$ can be written as $V_{0} A$ where $A \in \mathbb{R}^{\sigma(n, m)-r \times \sigma(n, m)-r}$ is an orthonormal matrix. Since it turns out that the eigenvalues of $A^{\prime} V_{0}^{\prime} L(\alpha) V_{0} A$ are the same of $V_{0}^{\prime} L(\alpha) V_{0}$, we can conclude that $\zeta\left(V_{0} A\right)=\zeta\left(V_{0}\right)$.

Theorem 1 provides a further necessary and sufficient condition to establish if a given matrix $G$ is a maximal SMR matrix. This condition is important because it states that the property of being a maximal SMR matrix is related only to the eigenspace of the minimum eigenvalue, contrary to the condition (11) which involves the whole matrix 
by exploiting the SOS index. Hence, Theorem 1 provides a way to construct maximal SMR matrices.

Observe that $\zeta\left(V_{0}\right)$ cannot be easily calculated because the set $\{\alpha$ : $\|\alpha\|=1\}$ is nonconvex. The following result proposes an alternative index for $V_{0}$.

Theorem 2: Let $w \in \mathbb{R}^{\tau(n, 2 m)}, w \neq 0$, be any vector and define

$$
\eta\left(V_{0}\right)=\max \left\{\eta\left(V_{0}, 1\right), \eta\left(V_{0},-1\right)\right\}
$$

where

$$
\eta\left(V_{0}, k\right)=\sup _{\alpha: w^{\prime} \alpha=k} \lambda_{\min }\left(V_{0}^{\prime} L(\alpha) V_{0}\right) .
$$

Then, $\zeta\left(V_{0}\right) \leq 0$ if and only if $\eta\left(V_{0}\right) \leq 0$. Moreover, $\eta\left(V_{0}\right)$ does not depend on the chosen decomposition of $G$.

Proof: " $\Rightarrow$ " Suppose for contradiction that $\zeta\left(V_{0}\right) \leq 0$ and $\eta\left(V_{0}\right)>0$. Then, there exists $\tilde{\alpha} \in \mathbb{R}^{\tau(n, 2 m)}$ such that $\left|w^{\prime} \tilde{\alpha}\right|=1$ and $\lambda_{\min }\left(V_{0}^{\prime} L(\tilde{\alpha}) V_{0}\right)>0$. Define $\bar{\alpha}=\|\tilde{\alpha}\|^{-1} \tilde{\alpha}$. We have that $\|\bar{\alpha}\|=1$ and $\lambda_{\min }\left(V_{0}^{\prime} L(\bar{\alpha}) V_{0}\right)=\|\tilde{\alpha}\|^{-1} \lambda_{\min }\left(V_{0}^{\prime} L(\tilde{\alpha}) V_{0}\right)>0$. This is impossible because $\zeta\left(V_{0}\right) \leq 0$.

" $\Leftarrow$ " Suppose for contradiction that $\eta\left(V_{0}\right) \leq 0$ and $\zeta\left(V_{0}\right)>0$. Then, there exists $\tilde{\alpha} \in \mathbb{R}^{\tau(n, 2 m)}$ such that $\|\tilde{\alpha}\|=1$ and $\lambda_{\min }\left(V_{0}^{\prime} L(\tilde{\alpha}) V_{0}\right)>0$. Suppose $w^{\prime} \tilde{\alpha} \neq 0$ and define $\bar{\alpha}=$ $\left|w^{\prime} \tilde{\alpha}\right|^{-1} \tilde{\alpha}$. We have that $\left\|w^{\prime} \bar{\alpha}\right\|=1$ and $\lambda_{\min }\left(V_{0}^{\prime} L(\bar{\alpha}) V_{0}\right)=$ $\left|w^{\prime} \tilde{\alpha}\right|^{-1} \lambda_{\min }\left(V_{0}^{\prime} L(\tilde{\alpha}) V_{0}\right)>0$. This is impossible because $\eta\left(V_{0}\right) \leq 0$. Suppose now that $w^{\prime} \tilde{\alpha}=0$. Then, for all $\varepsilon>0$ there exists $\hat{\alpha} \in \mathbb{R}^{\tau(n, 2 m)}$ such that $\|\hat{\alpha}\|=1$ and $\|\hat{\alpha}-\tilde{\alpha}\|<\varepsilon$ and $w^{\prime} \hat{\alpha} \neq 0$. For continuity of the function $\lambda_{\min }\left(V_{0}^{\prime} L(\alpha) V_{0}\right)$ with respect to $\alpha$, this $\hat{\alpha}$ can be chosen to satisfy also the constraint $\lambda_{\min }\left(V_{0}^{\prime} L(\hat{\alpha}) V_{0}\right)>0$. Repeating the procedure by using $\hat{\alpha}$ instead of $\tilde{\alpha}$, we conclude the proof.

Lastly, the choice of $V_{0}$ in the decomposition of $G$ does not affect $\eta\left(V_{0}\right)$ for the same reasoning of Theorem 1 .

Theorem 2 provides an alternative index for $V_{0}$ that can be computed through two convex optimizations. In fact, it turns out that $\eta\left(V_{0}, k\right)$ is the solution of the EVP

$$
\eta\left(V_{0}, k\right)=\sup _{t, \alpha} t \text { s.t. }\left\{\begin{array}{l}
w^{\prime} \alpha-k=0 \\
V_{0}^{\prime} L(\hat{\alpha}) V_{0}-t I_{\sigma(n, m)-r} \succeq 0 .
\end{array}\right.
$$

Observe that the free vector $w$ defines the two planes into which the unit shell $\{\alpha:\|\alpha\|=1\}$ used in Theorem 1 is crushed in order to achieve convexity. Although the sign of $\eta\left(V_{0}\right)$ does not depend on the choice of $w$, the absolute value does. Another difference between $\zeta\left(V_{0}\right)$ and $\eta\left(V_{0}\right)$ is that the former is bounded whereas the second may be not.

\section{B. PNS Characterization}

For $f(x) \in \Xi_{n, m}$ define the ball with radius $\delta \in \mathbb{R}$ centered in $f(x)$ as

$$
\mathcal{B}_{\delta}(f)=\left\{\tilde{f}(x) \in \Xi_{n, m}: d(\tilde{f}, f)<\delta\right\}
$$

where $d: \Xi_{n, m} \times \Xi_{n, m} \rightarrow \mathbb{R}$ is the distance in $\Xi_{n, m}$ defined as

$$
d(\tilde{f}, f)=\|\tilde{f}(x)-f(x)\|_{c} .
$$

Let us start by observing that, contrary to $\Xi_{n, 2 m}$ and $\Sigma_{n, 2 m}, \Delta_{n, 2 m}$ can be nonconvex. In fact, consider in $\Delta_{3,6}$ the Motzkin form and the Stengle form (see [23] and references therein)

$$
g_{\mathrm{Mot}}(x)=x_{1}^{4} x_{2}^{2}+x_{1}^{2} x_{2}^{4}+x_{3}^{6}-3 x_{1}^{2} x_{2}^{2} x_{3}^{2}
$$

$$
g_{\text {Ste }}(x)=x_{1}^{3} x_{3}^{3}+\left(x_{2}^{2} x_{3}-x_{1}^{3}-x_{1} x_{3}^{2}\right)^{2} .
$$

It can be verified that $\lambda\left(1 / 2\left(g_{\text {Mot }}+g_{\text {Ste }}\right)\right)=0$, that is $1 / 2\left(g_{\text {Mot }}(x)+\right.$ $\left.g_{\text {Ste }}(x)\right)$ is a SOS and not a PNS.

The following lemma introduces some remarks about the closeness between $\Delta_{n, 2 m}$ and $\Sigma_{n, 2 m}$.

Lemma 1: Suppose that $\Delta_{n, 2 m}$ is not empty. Then

a) there exists $g(x) \in \Delta_{n, 2 m}$ such that $\mu(g)>0$;

b) any $g(x) \in \Delta_{n, 2 m}$ such that $\mu(g)>0$ is an interior point of $\Delta_{n, 2 m}$, that is there exists $\delta>0$ such that $\mathcal{B}_{\delta}(g) \subset \Delta_{n, 2 m}$;

c) for all $g(x) \in \Delta_{n, 2 m}$ there exists $\delta>0$ such that $\mathcal{B}_{\delta}(g) \cap$ $\Phi_{n, 2 m} \subset \Delta_{n, 2 m}$.

Proof: First, if $\Delta_{n, 2 m}$ is not empty, there exists $g(x) \in \Delta_{n, 2 m}$ such that $\mu(g) \geq 0$. Suppose that $\mu(g)=0$ and define $\tilde{g}(x)=g(x)+$ $\varepsilon\|x\|^{2 m}$. It follows that $\mu(\tilde{g})=\mu(g)+\varepsilon=\varepsilon$. Moreover, from (6) we have that $G+\varepsilon I_{\sigma(n, m)}$ is a SMR matrix of $\tilde{g}(x)$. Hence, it follows that $\lambda(\tilde{g})=\lambda(g)+\varepsilon$. Since $\lambda(g)<0$ we conclude that, for all $0<\varepsilon<$ $-\lambda(g), \tilde{g}(x) \in \Delta_{n, 2 m}$ and $\mu(\tilde{g})>0$.

Second, consider $g(x) \in \Delta_{n, 2 m}$ such that $\mu(g)>0$. For continuity of $\mu(g)$ and $\lambda(g)$ with respect to $g(x)$, it follows that there exists $\delta>0$ such that, for all $\tilde{g}(x) \in \Xi_{n, 2 m}$ satisfying $\|\tilde{g}-g(x)\|_{c}<\delta, \mu(\tilde{g})>0$ and $\lambda(\tilde{g})<0$, that is $g(x)$ is an interior point of $\Delta_{n, 2 m}$.

Third, consider $g(x) \in \Delta_{n, 2 m}$. If $\mu(g)>0, g(x)$ is an interior point of $\Delta_{n, 2 m}$ and item 3) is clearly satisfied. Suppose hence $\mu(g)=0$. For the same reasoning of item 2), there exists $\delta>0$ such that, for all $\tilde{g}(x) \in \Xi_{n, 2 m}$ satisfying $\|\tilde{g}-g(x)\|_{c}<\delta, \lambda(\tilde{g})<0$, that is $\mathcal{B}_{\delta}(g) \cap \Sigma_{n, 2 m}=\emptyset$. Hence, item 3) holds.

Lemma 1 states that the set of PNS, if nonempty, contains form with a strictly positive positivity index, that is positive forms that vanish only in the origin. These forms are interior points for $\Delta_{n, 2 m}$, that is owning a neighborhood included in $\Delta_{n, 2 m}$. Moreover, it is stated that any PNS form owns a neighborhood where all positive forms are PNS, hence meaning that arbitrary small variations can not change a PNS into a SOS.

As we have seen in Section II-B, to establish whether a form $g(x)$ is a PNS amounts to establishing whether $\mu(g) \geq 0$ and $\lambda(g)<0$. The following result provides a further characterization of PNS and is the first step toward the construction of such forms.

Lemma 2: Let $G \in \mathcal{S}_{\sigma(n, m)}$ be any maximal SMR matrix of $g(x) \in$ $\Delta_{n, 2 m}$, and let $\left\langle\lambda_{\min }(G), \beta, V_{0}, V_{p}\right\rangle$ be any decomposition of $G$. Then

$$
\nexists x \neq 0: V_{p}^{\prime} x^{[m]}=0 \text {. }
$$

Proof: Now, suppose for contradiction that there exists $\tilde{x} \neq 0$ such that $\tilde{x}^{[m]} \in \operatorname{ker}\left(V_{p}^{\prime}\right)$. We have

$$
\begin{aligned}
g(\tilde{x})= & \tilde{x}^{[m]}\left[V_{0} V_{p}\right]\left(\lambda_{\min }(G) I_{\sigma(n, m)}\right. \\
& \left.+\left[\begin{array}{ll}
0 & \\
& \operatorname{diag}(\beta)
\end{array}\right]\right)\left[V_{0} V_{p}\right]^{\prime} \tilde{x}^{[m]} \\
= & \lambda_{\min }(G)\left\|V_{0}^{\prime} \tilde{x}^{[m]}\right\|^{2} .
\end{aligned}
$$

Observe that $\lambda_{\min }(G)<0$ since $G$ is a maximal SMR matrix of a PNS. Moreover, $\left\|V_{0}^{\prime} \tilde{x}^{[m]}\right\| \neq 0$ since $\operatorname{img}\left(V_{0}\right)=\operatorname{ker}\left(V_{p}^{\prime}\right)$. Hence, $g(\tilde{x})<0$. This is impossible because $g(x)$ is a PNS.

Lemma 2 provides a necessary condition for a form to be a PNS: the absence of solutions $x \neq 0$ in the polynomial system $V_{p}^{\prime} x^{[m]}=$ 0 . Observe that this condition is equivalent to the absence of vectors $x^{[m]} \neq 0$ in the linear space $\operatorname{img}\left(V_{0}\right)$.

The following result presents a way to generate PNS from any PNS. 
Theorem 3: Given $g(x) \in \Delta_{n, 2 m}$, let $G \in \mathcal{S}_{\sigma(n, m)}$ be any maximal SMR matrix of $g(x)$ and let $\left\langle\lambda_{\min }(G), \beta, V_{0}, V_{p}\right\rangle$ be any decomposition of $G$. For $\gamma \in \mathbb{R}^{r}, \gamma \geq 0$, define the SOS $s\left(x ; V_{p}, \gamma\right) \in \Sigma_{n, 2 m}$

$$
s\left(x ; V_{p}, \gamma\right)=x^{[m]} V_{p} \operatorname{diag}(\gamma) V_{p}^{\prime} x^{[m]} .
$$

and the cone of forms with vertex in $g(x)$

$$
\mathcal{C}(g)=\left\{h(x) \in \Xi_{n, 2 m}: h(x)=g(x)+s\left(x ; V_{p}, \gamma\right), \gamma \geq 0\right\} .
$$

Then, $\mathcal{C}(g) \subset \Delta_{n, 2 m}$. Moreover

$$
\exists \delta>0: \mu\left(g+s\left(V_{p}, \gamma\right)\right) \geq \mu(g)+\delta \min _{1 \leq i \leq r} \gamma_{i} .
$$

Proof: First of all, $s\left(x ; V_{p}, \gamma\right)$ is a SOS because its SMR matrix $S\left(V_{p}, \gamma\right)=V_{p} \operatorname{diag}(\gamma) V_{p}^{\prime}$ satisfies $S\left(V_{p}, \gamma\right) \succeq 0$ for all $\gamma \geq 0$.

In order to prove that $\mathcal{C}(g)$ contains only PNS, observe that $H=$ $G+S\left(V_{p}, \gamma\right)$ is a maximal SMR matrix of $h(x)=g(x)+s\left(x ; V_{p}, \gamma\right)$. In fact

$$
\begin{aligned}
H= & {\left[V_{0} V_{p}\right]\left(\lambda_{\min }(G) I_{\sigma(n, m)}\right.} \\
& \left.+\left[\begin{array}{rr}
0 & \\
& \operatorname{diag}(\beta)
\end{array}\right]\right)\left[V_{0} V_{p}\right]^{\prime}+V_{p} \operatorname{diag}(\gamma) V_{p}^{\prime} \\
= & {\left[V_{0} V_{p}\right]\left(\lambda_{\min }(G) I_{\sigma(n, m)}+\left[\begin{array}{c}
0 \\
\operatorname{diag}(\beta+\gamma)
\end{array}\right]\right)\left[V_{0} V_{p}\right]^{\prime} }
\end{aligned}
$$

which clearly implies that $\left\langle\lambda_{\min }(G), \beta+\gamma, V_{0}, V_{p}\right\rangle$ is a decomposition of $H$. Hence, from Theorem 1 it follows that $H$ is a maximal SMR matrix because $\zeta\left(V_{0}\right) \leq 0$ being $G$ a maximal SMR matrix. From the fact that $H$ is a maximal SMR matrix it follows that $\lambda(h)=$ $\lambda_{\min }(H)=\lambda_{\min }(G)=\lambda(g)$. Moreover, we have that $\mu(h) \geq \mu(g)$ because $s\left(x ; V_{p}, \gamma\right)$ is a SOS. Since $g(x) \in \Delta_{n, 2 m}$ we conclude that $\lambda(h)=\lambda(g)<0$ and $\mu(h) \geq \mu(g) \geq 0$, that is $h(x) \in \Delta_{n, 2 m}$.

Lastly, observe that $\mu\left(g+s\left(V_{p}, \gamma\right)\right) \geq \mu(g)+\mu\left(s\left(V_{p}, \gamma\right)\right)$. Moreover,

$$
s\left(x ; V_{p}, \gamma\right) \geq\left\|V_{p}^{\prime} x^{[m]}\right\|^{2} \min _{1 \leq i \leq r} \gamma_{i} \quad \forall x \forall \gamma
$$

According to Lemma $2, V_{p}^{\prime} x^{[m]} \neq 0$ for all $x \neq 0$. Hence, (31) holds with $\delta=\mu(v)>0$ where $v(x)=\left\|V_{p}^{\prime} x^{[m]}\right\|^{2}$.

Theorem 3 states that any PNS is the vertex of a cone of PNS. In particular, the cone is unbounded and its directions correspond to strictly positive SOS that can be linearly parameterized in a convex set. Observe also that, according to (31), there exist PNS whose positivity index $\mu$ is arbitrarily large, that is arbitrarily large positive forms that are not SOS.

How to construct PNS? In order to answer to this question, let us define the set

$$
\Theta_{n, 2 m}=\bigcup_{1 \leq r \leq \sigma(n, m)} \Theta_{n, 2 m}(r)
$$

where

$$
\begin{gathered}
\Theta_{n, 2 m}(r)=\left\{\left\langle\delta, \beta, V_{p}\right\rangle: \delta \in \mathbb{R}, \delta \in(0,1],\right. \\
\left.\beta \in \mathbb{R}^{r}, \beta>0, \quad \text { and } V_{p} \in \mathcal{V}_{n, 2 m}(r)\right\} \\
\mathcal{V}_{n, 2 m}(r)=\left\{V_{p} \in \mathbb{R}^{\sigma(n, m) \times r}: V_{p}^{\prime} V_{p}=I_{r},\right. \\
\left.\zeta\left(\operatorname{cmp}\left(V_{p}\right)\right) \leq 0, \text { and (28)holds }\right\}
\end{gathered}
$$

and $\operatorname{cmp}\left(V_{p}\right) \in \mathbb{R}^{\sigma(n, m) \times \sigma(n, m)-r}$ is a matrix whose columns are an orthonormal base of $\operatorname{ker}\left(V_{p}^{\prime}\right)$. For $\theta \in \Theta_{n, 2 m}(r)$ define the form

$$
\psi(x ; \theta)=s\left(x ; V_{p}, \beta\right)-\delta \mu\left(s\left(V_{p}, \beta\right)\right)\|x\|^{2 m} .
$$

The following result provides an answer to the question introducing a parameterization of $\Delta_{n, 2 m}$.

Theorem 4: For all $g(x) \in \Delta_{n, 2 m}$, there exists $\theta \in \Theta_{n, 2 m}$ such that $g(x)=\psi(x ; \theta)$. Moreover, $\psi(x ; \theta) \in \Delta_{n, 2 m}$ for all $\theta \in \Theta_{n, 2 m}$.

Proof: Suppose $g(x) \in \Delta_{n, 2 m}$. Let $G$ be a maximal SMR matrix of $g(x)$, and let $\left\langle\lambda_{\min }(G), \beta, V_{0}, V_{p}\right\rangle$ be a decomposition of $G$. We have:

$$
\begin{aligned}
g(x)= & x^{[m]}\left[V_{0} V_{p}\right]\left(\lambda_{\min }(G) I_{\sigma(n, m)}\right. \\
& \left.+\left[\begin{array}{rr}
0 & \\
& \operatorname{diag}(\beta)
\end{array}\right]\right)\left[V_{0} V_{p}\right]^{\prime} x^{[m]} \\
= & x^{[m]}\left(\lambda_{\min }(G) I_{\sigma(n, m)}+V_{p} \operatorname{diag}(\beta) V_{p}^{\prime}\right) x^{[m]} \\
= & \lambda_{\min }(G)\|x\|^{2 m}+s\left(x ; V_{p}, \beta\right) .
\end{aligned}
$$

Hence, $g(x)=\psi(x ; \theta)$ where $\theta=\left\langle\delta, \beta, V_{p}\right\rangle$ and

$$
\delta=-\frac{\lambda_{\min }(G)}{\mu\left(s\left(V_{p}, \beta\right)\right)} .
$$

Observe that $\delta \in(0,1]$ because $\lambda_{\min }(G)=\lambda(g)<0$ and $\lambda_{\min }(G)+$ $\mu\left(s\left(V_{p}, \beta\right)\right)=\mu(g) \geq 0$. Moreover, $\beta>0$. Then, from Theorem 1 and Lemma 2, it follows that $V_{p} \in \mathcal{V}_{n, 2 m}(r)$ where $r$ is the length of $\beta$. Therefore, $\theta \in \Theta_{n, 2 m}$.

Now, consider $\theta=\left\langle\delta, \beta, V_{p}\right\rangle \in \Theta_{n, 2 m}$. We have that a SMR matrix of $\psi(x ; \theta)$ is given by

$$
\begin{aligned}
\Psi(\theta)= & V_{p} \operatorname{diag}(\beta) V_{p}^{\prime}-\delta \mu\left(s\left(V_{p}, \beta\right)\right) I_{\sigma(n, m)} \\
= & {\left[\operatorname{cmp}\left(V_{p}\right) V_{p}\right]\left(\left[\begin{array}{lr}
0 & \\
& \operatorname{diag}(\beta)
\end{array}\right]\right.} \\
& \left.-\delta \mu\left(s\left(V_{p}, \beta\right)\right) I_{\sigma(n, m)}\right)\left[\operatorname{cmp}\left(V_{p}\right) V_{p}\right]^{\prime} .
\end{aligned}
$$

Since $V_{p}^{\prime} V_{p}=I_{r}$ and $\beta>0$, it follows that $\left\langle-\delta \mu\left(s\left(V_{p}, \beta\right)\right), \beta, \operatorname{cmp}\left(V_{p}\right), V_{p}\right\rangle \quad$ is a decomposition of $\Psi(\theta)$. From Theorem 1 we have that $\Psi(\theta)$ is a maximal SMR matrix because $\zeta\left(\operatorname{cmp}\left(V_{p}\right)\right) \leq 0$. Moreover, from Lemma 2 it follows that $\mu\left(s\left(V_{p}, \beta\right)\right)>0$. Hence, $\lambda(\psi(\theta))=-\delta \mu\left(s\left(V_{p}, \beta\right)\right)<0$ and $\mu(\psi(\theta))=(1-\delta) \mu\left(s\left(V_{p}, \beta\right)\right) \geq 0$. Therefore, $\psi(x ; \theta) \in \Delta_{n, 2 m} . \square$

Theorem 4 states that the set of PNS is the image of $\Theta_{n, 2 m}$ through the function $\psi(x ; \theta)$. This result provides hence a technique to construct all existing PNS that amounts to finding matrices $V_{p}$ in $\mathcal{V}_{n, 2 m}(r)$ and calculating the positivity index $\mu\left(s\left(V_{p}, \beta\right)\right)$.

Unfortunately, the set $\mathcal{V}_{n, 2 m}(r)$ can not be explicitly described at present. A method to find elements in this set consists of looking for matrices $V_{p}$ with a fixed structure for which the property (28) and the positivity index $\mu\left(s\left(V_{p}, \beta\right)\right)$ can be easily assessed, and using the remaining free parameters to satisfy $\zeta\left(\operatorname{cmp}\left(V_{p}\right)\right) \leq 0$.

\section{ILLUSTRATIVE EXAMPLES}

\section{A. Example 1}

Let us consider the Stengle form in (27). Let us obtain a maximal SMR matrix $G$ of this form as done in (12) by selecting

$$
\begin{aligned}
x^{[m]}= & {\left[x_{1}^{3}, \sqrt{3} x_{1}^{2} x_{2}, \sqrt{3} x_{1}^{2} x_{3}, \sqrt{3} x_{1} x_{2}^{2}\right.} \\
& \left.\sqrt{6} x_{1} x_{2} x_{3}, \sqrt{3} x_{1} x_{3}^{2}, x_{2}^{3}, \sqrt{3} x_{2}^{2} x_{3}, \sqrt{3} x_{2} x_{3}^{2}, x_{3}^{3}\right]^{\prime}
\end{aligned}
$$


and $L(\alpha)$ as the matrix returned by the algorithm in [20], which is a matrix in $\mathcal{S}_{10}$ depending linearly on $\alpha \in \mathbb{R}^{27}$. Let us compute a decomposition $\left\langle\lambda_{\min }(G), \beta, V_{0}, V_{p}\right\rangle$ of this maximal SMR matrix according to (13)-(15) via simple eigenvalue and eigenvector computation. We find that $V_{p}^{\prime} x^{[m]}=\left[w_{1}(x), w_{2}(x), w_{3}(x)\right]^{\prime}$ with $w_{1}(x), w_{2}(x), w_{3}(x)$ as shown in the equation at the bottom of the page. Hence, from Theorem 3 one has that

$$
g_{\mathrm{Ste}}(x)+\sum_{i=1}^{3} \gamma_{i} w_{i}(x)^{2}
$$

is a PNS for all $\gamma \geq 0$, which can be used as a Lyapunov function candidate in stability and performance problems.

\section{B. Example 2}

We show here the construction of a simple PNS by using Theorem 4 with $n=m=3$. Choose $x^{[m]}$ and $L(\alpha)$ as in Example 1. Select $r=3$ and

$$
V_{p}=\frac{1}{7}\left[\begin{array}{cccccccccc}
6 & 0 & 0 & -2 & 0 & -3 & 0 & 0 & 0 & 0 \\
0 & -3 & 0 & 0 & 0 & 0 & 6 & 0 & -2 & 0 \\
0 & 0 & -2 & 0 & 0 & 0 & 0 & -3 & 0 & 6
\end{array}\right]^{\prime}
$$

Observe that $V_{p}^{\prime} V_{p}=I_{r}$. Moreover, we find $\eta\left(\operatorname{cmp}\left(V_{p}\right)\right)=-0.041$ (by selecting $w=[1,0, \cdots, 0]^{\prime}$ in (22)) which allows us to conclude from Theorem 2 that $\zeta\left(\operatorname{cmp}\left(V_{p}\right)\right)<0$. Now, the structure of $V_{p}$ allows us to easily assess the property (28) and the positivity index $\mu\left(s\left(V_{p}, \beta\right)\right)$. In fact, $V_{p}^{\prime} x^{[m]}=1 / 7\left[w_{1}(x), w_{2}(x), w_{3}(x)\right]^{\prime}$ where

$$
\left\{\begin{array}{l}
w_{1}(x)=x_{1}\left(6 x_{1}^{2}-2 \sqrt{3} x_{2}^{2}-3 \sqrt{3} x_{3}^{2}\right) \\
w_{2}(x)=x_{2}\left(-3 \sqrt{3} x_{1}^{2}+6 x_{2}^{2}-2 \sqrt{3} x_{3}^{2}\right) \\
w_{3}(x)=x_{3}\left(-2 \sqrt{3} x_{1}^{2}-3 \sqrt{3} x_{2}^{2}+6 x_{3}^{2}\right)
\end{array}\right.
$$

It is straightforward to see that $w_{1}(x)=w_{2}(x)=w_{3}(x)=0$ if and only if $x=0$, that is (28) holds. Hence, $V_{p} \in \mathcal{V}_{n, 2 m}(r)$ and

$$
\theta=\left\langle\delta, \beta, V_{p}\right\rangle \in \Theta_{n, 2 m} \quad \forall \delta \in(0,1] \forall \beta>0 .
$$

Select $\beta=[49,49,49]^{\prime}$. We have that

$$
s\left(x ; V_{p}, \beta\right)=w_{1}(x)^{2}+w_{2}(x)^{2}+w_{3}(x)^{2} .
$$

In order to compute $\mu\left(s\left(V_{p}, \beta\right)\right)$, one has to find the minimum of $s\left(x ; V_{p}, \beta\right)$ subject to $\|x\|=1$. Let us observe that, since $s\left(x ; V_{p}, \beta\right)$ depends directly on $x_{1}^{2}, x_{2}^{2}, x_{3}^{2}$, one can first substitute $x_{3}^{2}=1-x_{1}^{2}-x_{2}^{2}$ in $s\left(x ; V_{p}, \beta\right)$, and then find the minimum by computing the points where the derivatives of $s\left(x ; V_{p}, \beta\right)$ with respect to $x_{1}^{2}$ and $x_{2}^{2}$ vanish. This operation amounts to solving a system of two quadratic equations in two variables, and can be done by finding the roots of a polynomial equation of degree four in one variable via the resultants method. We find $\mu\left(s\left(V_{p}, \beta\right)\right)=0.7433$. Therefore, from Theorem 4 , it follows that

$$
\psi(x ; \theta)=w_{1}(x)^{2}+w_{2}(x)^{2}+w_{3}(x)^{2}-0.7433\|x\|^{2 m} \delta
$$

is a PNS for all $\delta \in(0,1]$.

Lastly, from Theorem 3 it follows that the cone

$$
\begin{aligned}
\mathcal{C}(\psi(\theta)) & \\
= & \left\{h(x) \in \Xi_{n, 2 m}: h(x)=\psi(x ; \theta)+s\left(x ; V_{p}, \gamma\right), \gamma \geq 0\right\} \\
= & \left\{h(x) \in \Xi_{n, 2 m}: h(x)=\sum_{i=1}^{3}\left(1+\gamma_{i}\right) w_{i}(x)^{2}\right. \\
& \left.-0.7433\|x\|^{2 m} \delta, \gamma \geq 0\right\}
\end{aligned}
$$

with vertex $\psi(x ; \theta)$ contains only PNS, that is $\mathcal{C}(\psi(\theta)) \subset \Delta_{n, 2 m}$.

\section{CONCLUSION}

The gap existing between positive polynomials and SOS of polynomials has been investigated in this paper by proposing a matrix characterization of the PNS, that is the homogeneous forms that are not SOS. This characterization is based on eigenvectors and eigenvalues decomposition, and provides new results about the structure of these forms. In particular, it is shown that any PNS is the vertex of an unbounded cone of PNS. Moreover, a complete parametrization of the set of PNS is introduced which allows one to construct PNS.

These results can allow one to achieve less conservative results in analysis and synthesis problems by providing new Lyapunov function candidates which are not SOS. Moreover, it is expected that the proposed characterization play a significant role in future investigations of this gap which affects several analysis and synthesis tools in control systems and about which almost nothing is known.

\section{ACKNOWLEDGMENT}

This work was partially done when the author was with the Department of Information Engineering of the University of Siena. The author would like to thank the Associate Editor and the Reviewers for their time and constructive comments.

\section{REFERENCES}

[1] G. Chesi, A. Tesi, A. Vicino, and R. Genesio, "On convexification of some minimum distance problems," in Proc. 5th European Contr. Conf., Karlsruhe, Germany, 1999.

[2] S. Boyd, L. El. Ghaoui, E. Feron, and V. Balakrishnan, Linear Matrix Inequalities in System and Control Theory. Philadelphia, PA: SIAM, 1994.

[3] A. L. Zelentsovsky, "Nonquadratic Lyapunov functions for robust stability analysis of linear uncertain systems," IEEE Trans. Autom. Control, vol. 39, no. 1, pp. 135-138, 1994.

[4] P.-A. Bliman, "Stabilization of LPV systems," in Proc. 42st IEEE Conf. Dec. Contr., Maui, HI, 2003, pp. 6103-6108.

$$
\left\{\begin{array}{l}
w_{1}(x)=-0.503 x_{1}^{2} x_{2}+2.335 x_{1} x_{2} x_{3}+0.013 x_{2}^{3}-0.147 x_{2} x_{3}^{2} \\
w_{2}(x)=-0.455 x_{1}^{3}+1.247 x_{1}^{2} x_{3}+0.067 x_{1} x_{2}^{2}+0.0833 x_{1} x_{3}^{2}-0.355 x_{2}^{2} x_{3}-0.007 x_{3}^{3} \\
w_{3}(x)=-0.782 x_{1}^{3}-0.152 x_{1}^{2} x_{3}+0.004 x_{1} x_{2}^{2}-0.716 x_{1} x_{3}^{2}+0.793 x_{2}^{2} x_{3}-0.002 x_{3}^{3} .
\end{array}\right.
$$


[5] G. Chesi, A. Garulli, A. Tesi, and A. Vicino, "Robust stability of time-varying polytopic systems via parameter-dependent homogeneous Lyapunov functions," Automatica, vol. 43, no. 2, pp. 309-316, 2007.

[6] R. Goebel, A. R. Teel, T. Hu, and Z. Lin, "Dissipativity for dual linear differential inclusions through conjugate storage functions," in Proc. 43rd IEEE Conf. Dec. Contr., Paradise Island, Bahamas, 2004.

[7] A. Trofino and C. E. D. Souza, "Bi-quadratic stability of uncertain linear systems," in Proc. 38th IEEE Conf. Dec. Contr., Phoenix, AZ, 1999.

[8] G. Chesi, A. Garulli, A. Tesi, and A. Vicino, "Polynomially parameter-dependent Lyapunov functions for robust stability of polytopic systems: An LMI approach," IEEE Trans. Autom. Control, vol. 50, no. 3, pp. 365-370, 2005.

[9] P.-A. Bliman, "A convex approach to robust stability for linear systems with uncertain scalar parameters," SIAM J. Contr. Optim., vol. 42, no. 6, pp. 2016-2042, 2004.

[10] C. Hol and C. Scherer, "Computing optimal fixed order $\mathcal{H}_{\infty}$-synthesis values by matrix sum of squares relaxations," in Proc. 43rd IEEE Conf. Dec. Contr., Paradise Island, Bahamas, 2004.

[11] G. Chesi, A. Garulli, A. Tesi, and A. Vicino, "Polynomially parameter-dependent Lyapunov functions for robust $\mathcal{H}_{\infty}$ performance analysis," in Proc. 16th IFAC World Congr. Autom. Control, Prague, Czech Republic, 2005.

[12] B. Tibken, "Estimation of the domain of attraction for polynomial systems via LMI's," in Proc. 39th IEEE Conf. Dec. Contr., Sydney, Australia, 2000, pp. 3860-3864.

[13] S. Prajna, P. A. Parrilo, and A. Rantzer, "Nonlinear control synthesis by convex optimization," IEEE Trans. Autom. Control, vol. 49, no. 2 , pp. 310-314, 2004.

[14] S. Prajna, A. Papachristodoulou, and F. Wu, "Nonlinear control synthesis by sum of squares optimization: A Lyapunov-based approach," in Proc. Asian Contr. Conf., 2004.

[15] G. Chesi, "Computing output feedback controllers to enlarge the domain of attraction in polynomial systems," IEEE Trans. Autom. Control, vol. 49, no. 10, pp. 1846-1850, 2004.

[16] S. Prajna and A. Papachristodoulou, "Analysis of switched and hybrid systems-beyond piecewise quadratic methods," in Proc. Amer. Contr. Conf., Denver, CO, 2003.

[17] S. Prajna and A. Jadbabaie, "Safety verification of hybrid systems using barrier certificates," in Hybrid Systems: Computation and Control, R. Alur and G. J. Pappas, Eds. New York: Springer-Verlag, 2004.

[18] A. Papachristodoulou, "Analysis of nonlinear time delay systems using the sum of squares decomposition," in Proc. Amer. Contr. Conf., Boston, MA, 2004.

[19] P. A. Parrilo, "Structured Semidefinite Programs and Semialgebraic Geometry Methods in Robustness and Optimization," Ph.D. dissertation, Control and Dynamical Systems, California Inst. Technol., Pasadena, CA, 2000.

[20] G. Chesi, A. Garulli, A. Tesi, and A. Vicino, "Solving quadratic distance problems: An LMI-based approach," IEEE Trans. Autom. Control, vol. 48, no. 2, pp. 200-212, 2003.

[21] Z. Jarvis-Wloszek, R. Feeley, W. Tan, K. Sun, and A. Packard, "Some controls applications of sum of squares programming," in Proc. 42nd IEEE Conf. Dec. Contr., Maui, HI, 2003, pp. 4676-4681.

[22] D. Henrion and A. Garulli, Eds., "Positive Polynomials in Control," in Ser. Lecture Notes in Control and Information Sciences. London, U.K.: Springer-Verlag, 2005, no. 312.

[23] B. Reznick, "Some concrete aspects of hilbert's 17th problem," Contemp. Math., vol. 253, pp. 251-272, 2000.

[24] G. Hardy, J. E. Littlewood, and G. Pólya, Inequalities: Second Edition. Cambridge, U.K.: Cambridge University Press, 1988.

\section{Stability Analysis of a Class of PWM Systems}

Stefan Almér, Ulf Jönsson, Chung-Yao Kao, and Jorge Mari

Abstract-This note considers stability analysis of a class of pulsewidth modulated (PWM) systems that incorporates several different switched mode dc-dc converters. The systems of the class typically have periodic solutions. A sampled data model is developed and used to prove stability of these solutions. Conditions for global and local exponential stability are derived using quadratic and piecewise quadratic Lyapunov functions. The state space is partitioned and the stability conditions are verified by checking a set of coupled linear matrix inequalities (LMIs).

Index Terms-dc-dc converter, Lyapunov methods, pulsewidth modulated (PWM) systems, sampled data modeling, stability analysis.

\section{INTRODUCTION}

This note presents a method for stability analysis of a class of pulsewidth modulated (PWM) systems. The systems switch periodically between two affine vector fields to create a periodic solution at stationarity. The only control variable is the so called duty ratio which determines the fraction of time each vector field is active.

Our motivation for the analysis comes mainly from switched mode dc-dc converters [1] which are used extensively in power supplies of various electronic circuits. However, PWM systems are found in a wide range of applications, ranging from power conversion to hydraulic systems.

Conventionally, dc-dc converters are controlled using analog PWM techniques that rely on a comparator ramp function. In this note we consider a switching technique referred to as digital PWM where the switching is based on the sampled state. Digital PWM offers advantages such as being less sensitive to noise and aging of components and has received much attention recently, see e.g., [2]. It should be noted that analog PWM can also be treated in our framework. (See [3] for a detailed description.)

Much of the reported analysis on PWM systems is based on the averaging approach [4], [5]. However, averaging is only an approximation of the low frequency system dynamics and it requires sufficiently high switching frequency to be adequate. Furthermore, in many applications the averaged model will be nonlinear and difficult to analyze. The contribution of this note is to provide a systematic method for stability analysis which does not resort to averaging or linearization.

Our starting point is a stationary periodic solution and we proceed to derive criteria for stability and uniqueness of such a solution. The

Manuscript received May 6, 2005; revised November 7, 2006. Recommended by Associate Editor L. Glielmo. This work was supported by the Swedish Research Council, the Gustafsson Foundation, the Australian Research Council and the HYCON Network of Excellence, E.C. Grant IST-511368.

S. Almér is with the Division of Optimization and Systems Theory, Department of Mathematics, Royal Institute of Technology, 10044 Stockholm, Sweden (e-mail: almer@math.kth.se).

U. Jönsson is with the Division of Optimization and Systems Theory, Department of Mathematics, Royal Institute of Technology, 10044 Stockholm, Sweden (e-mail: ulfj@math.kth.se).

C.-Y. Kao is with the Department of Electrical and Electronic Engineering, University of Melbourne, Parkville 3010 VIC, Australia (e-mail: cykao@ee.mu.oz.au).

J. Mari is with the Electrical Energy Systems, GE Global Research, D-85748 Munchen, Germany.

Color versions of one or more of the figures in this paper are available online at http://ieeexplore.ieee.org.

Digital Object Identifier 10.1109/TAC.2007.899082 